\title{
ESTILOS PARENTALES DE ENSEÑANZA EN FAMILIAS CON NIÑOS CON NECESIDADES EDUCATIVAS ESPECIALES EN SAMACÁ-BOYACÁ
}

\author{
TEACHING PARENTING STYLES IN FAMILIES WITH CHILDREN WITH SPECIAL NEEDS \\ IN SAMACÁ- BOYACÁ
}

Julio Fernando Acosta-Muñoz*, Ana milena Toro-Ibarra** y Maira Alejandra Guerrero-Ramírez***

\begin{abstract}
RESUMEN
El presente artículo de investigación surge de la pesquisa denominada "Estilos parentales de enseñanza en familias con niños con necesidades educativas especiales de Samacá", cuyo objetivo es indagar los estilos parentales de enseñanza en las familias con niños que presentan necesidades educativas especiales en la Institución Educativa La Libertad del municipio de Samacá en Boyacá, a través de la metodología de la investigación cualitativa, con un enfoque crítico-social partiendo del método de la etnografía crítica. La población para el presente estudio estuvo compuesta por 15 familias del municipio. La estrategia de recolección de información se basó en la aplicación de entrevista, relatos de vidas, talleres investigativos y diarios de campo. El análisis de resultados se realizó desde el sistema categorial, el cual permitió ver que los estilos parentales de enseñanza de la población están establecidos como consecuencia de factores culturales y a partir de las experiencias vividas de los padres en su infancia, además se evidenció que los padres no comparten un mismo estilo parental, puesto que la figura paterna muestra negligencia en el cuidado y educación de sus hijos, mientras que la figura materna se considera la única responsable del cuidado de los hijos y de las demás responsabilidades del hogar.
\end{abstract}

Palabras clave. Estilos parentales de enseñanza, necesidades educativas especiales, familia.

\begin{abstract}
The current investigation had as an objective to inquire about the parental teaching styles in families with children that show special educative needs, which assist to "Institución Educativa La Libertad" in Samacá-Boyacá, trough methodology of qualitative investigation, with a critic-social focus starting off the method of critic ethnography. The population for the current research was composed by fifteen families of the municipality. The information collection strategy was based in the application of interviews, life's reports, investigative workshops and field diaries. The result analysis was performed from the categorical
\end{abstract}

Fecha de recepción: Febrero 23 de 2015 / Fecha de aceptación: Abril 07 de 2015

Tipología: Artículo de Investigación Científica y Tecnológica

Para citar este artículo: Acosta, M. J., Toro, I. A., \& Guerrero, R. M. (2015). Estilos parentales de enseñanza en familias con niños con necesidades educativas especiales en Samacá-Boyacá. Praxis. Vol. 11, 19 - 29

\footnotetext{
*Magister en Educación, Universidad Pedagógica y Tecnológica de Colombia, Colombia. Email: jufeacmu@hotmail.com

**Estudiante, Universidad Pedagógica y Tecnológica de Colombia, Colombia.Email: anelim6886@hotmail.com

***Estudiante, Universidad Pedagógica y Tecnológica de Colombia, Colombia.Email: malejaguerrero@outlook.es
} 
system, which allowed seeing that the parental teaching styles in population are established as a consequence of cultural factors and lived experiences of parents in their childhood, besides was evidenced that parents don't share a same parental style, because the paternal figure shows negligence in the care and education of its children, while the maternal figure is considered the only responsible of the care of children and other responsibilities of home.

Key words. Teaching parenting styles, special needs, family.

\section{INTRODUCCIÓN}

$\mathrm{D}$ urante las últimas décadas se han multiplicado las investigaciones sobre los estilos y las prácticas educativas de los padres, así como su influencia en el desarrollo de los niños en los diferentes ambientes en los que éstos crecen (Borke, Lamm, Eickhorst \& Keller, 2007). La familia se concibe como una estructura básica conformada por diferentes personas que conviven de manera prolongada y que desempeñan diferentes roles y actitudes, según sea su desenvolvimiento socio-cultural. Así mismo, la crianza en los niños como parte de los roles culturales de la familia, se vuelve parte fundamental para su crecimiento y desarrollo como personas: la crianza hace parte de las relaciones familiares y en ellas se resalta el papel que juegan los padres en la formación de sus hijos (Myers, 1994; Aguirre, 2000). Las familias a través de los estilos parentales utilizados pueden comunicar a sus hijos las diferentes exigencias sobre las actividades diarias, tales como comportamientos, actitudes, dedicación, responsabilidad frente a sus obligaciones. El sistema familiar juega un papel importante en la formación de los individuos especialmente en los primeros años, debido que el tipo de pautas que se desarrollan en el núcleo familiar generan e imprimen en los niños una serie de herramientas que les permiten constituir formas de comportamiento social, que se reflejará en la comunión con los otros. Los estilos parentales de enseñanza que utilizan los padres con sus hijos pueden generar en estos efectos inmediatos o duraderos, en su desarrollo social, moral, afectivo, familiar y académico (Ceballos \& Rodrigo, 1998).

La educación y las pautas de crianza en el núcleo familiar con niños con necesidades educativas espaciales, al igual que cualquier tipo de educación instruida a este tipo de niños, presenta una serie de debilidades. Según las indagaciones de Reynaud y Sánchez (1996), los límites bajo los cuales los padres de familia con niños con dificultades cognitivas, operan en el uso de técnicas de control directo, es decir, ellos son los que resuelven los problemas e imponen soluciones a sus hijos, considerando que sus hijos deben obedecer a una serie de reglas absolutas y esperan de ellos obediencia y respeto a la autoridad permitiendo poca independencia; el otro extremo de padres permiten que sus hijos actúen impulsivamente, castigan poco y no prohíben, no demandan una conducta madura por parte de sus hijos y no establecen límites o reglas.

Según Baumrind (1966), las pautas de crianza que se asumen en alguno de los ambos extremos anteriormente mencionados, podrían llegar a generar comportamientos inadecuados en el núcleo familiar con este tipo de niños, por lo que se hace relevante que los padres conozcan acerca de la importancia que ellos ejercen en el desarrollo de sus hijos. Este prototipo de enseñanza parental en los niños con necesidades educativas especiales, ante las dificultades mencionadas, lleva en muchos casos a tratar de compensar la falta de afecto con estímulos materiales, además que los criados bajo estos modelos, pueden presentar un auto-concepto negativo, problemas de autoconfianza, irresponsabilidad, trastornos afectivos, problemas de conducta e irrespeto frente a las figuras de autoridad.

Los niños con necesidades educativas especiales requieren de atención, apoyo, comprensión, paciencia y dedicación para promover en ellos seguridad y confianza al momento de realizar sus actividades familiares, sociales y académica. 
Como plantea Aranda (2009), un niño con necesidades educativas especiales debe ser comprendido y aceptado por sus padres y demás miembros de la familia, pues en ello se inicia la educación y es el marco propicio para su desarrollo personal. Este tipo de atención y apoyo primario a este tipo de población, se ve distorsionado y disminuido en el momento en que los padres de familia que en el afán de desarrollar pautas de crianza asertivas, desconocen las características emocionales, cognitivas y afectivas de este tipo de niños, asociado a la inexperiencia y ausencia de estrategias para el trato adecuado y asertivo de la educación familiar en este tipo de niños, utilizando en muchos casos el castigo en todas sus formas, como mecanismo para educar las acciones o comportamientos diferentes de estos niños.

En la investigación desarrollada por Ávila, Malagón y Triana (2010), en 78 municipios en el departamento de Boyacá, se evidencia que los estilos parentales en nuestro departamento se caracterizan en la predominancia de un estilo parental patriarcal-machista, donde los patrones de crianza en gran parte es oficio de la madre, ésta es la encargada de la totalidad de las labores domésticas y parte de las agropecuarias. La mayor parte del tiempo los niños y niñas comparten el tiempo con las madres, lo que lleva a que ellos sean vinculados a estas actividades y se vuelvan parte de sus obligaciones diarias, sumadas a las demás labores del infante (escolares, sociales, etc.). Una de las dificultades más sentidas que se presentan frente a este tema en el área rural, es que los padres centran más la atención en el trabajo del campo que en las actividades del hogar, confiando la educación y la crianza de sus hijos a los docentes, porque en muchos casos, los padres de familia no cuentan con la formación necesaria para brindar apoyo a sus hijos desde el hogar.

Bajo este panorama, se puede evidenciar que una de las características en la educación familiar de los menores de edad en Boyacá, está caracterizada por la ausencia de los padres en las actividades diarias y desarrollo psicosocial de los niños, además es importante resaltar que en algunos casos se ve la presencia de segundos cuidadores, que intentan reemplazar el rol de los padres, en estos casos esos segundo cuidadores son los profesores, madres comunitarias, abuelos, hermanos mayores y vecinos (Ávila, Malagón, \& Triana, 2010). Los estilos parentales de enseñanza que se asuman en el núcleo familiar tienen una directa afectación sobre la formación de los niños, estilos que dependen y partiendo del punto extremo que asuman los padres, consolidan la forma en que los infantes se relacionan con el entorno. Ese tipo de condiciones que se generan dentro del ámbito familiar, se evidencian en cualquier contexto, estrato o cultura donde este inmerso el núcleo familiar.

Las familias con niños con necesidades educativas especiales en Boyacá no está exenta de manifestar las anteriores dificultades relacionadas con el estilo parental de enseñanza asumido. Sin embargo existe la ausencia empírica que pueda sustentar estas dificultades en la población núcleo de esta investigación. Es por esto que surge la necesidad de indagar cómo son los estilos parentales en los núcleos familiares con este tipo de población, con el fin de no solo identificar las dificultades frente a este tema, sino evidenciar también las posibles fortalezas que se pueden desarrollar frente a este tipo de población, a través de un estudio riguroso basado en la etnografía crítica. Cabe también señalar que los antecedentes empíricos frente al tema de investigación son escasos, además que la evidencia investigativa frente a la identificación del contenido de los estilos parentales de enseñanza en familias con necesidades educativas espaciales en el área rural, sistematizados y debidamente publicados en revistas de investigación, desde el rastreo en distintas bases de datos en nacionales e internacionales no existe, y menos aún en el contexto rural en el departamento de Boyacá.

La significatividad de esta pesquisa está en constituir una caracterización sobre los estilos parentales en familias con niños con necesidades educativas especiales en el área rural, dada en la descripción y análisis de los distintos contenidos, diálogos y contextos donde se desarrollan los estilos parentales de enseñanza, sumado a la particularidad cultural de estas familias, además de ser una forma de entender la estructura 
social y educativa de los hogares rurales y sus integrantes: es recorrer cada ensamble, encontrar lo dinámico y lo estático, estructurar descripciones para proyectar el cambio que haga posible la construcción de nuevas relaciones familiares en el entorno rural. A través del encuentro de los criterios y los juicios hechos por el grupo de padres de familia participantes en esta investigación, se podrá demarcar, caracterizar, escrutar y darle sentido a esa realidad experiencial, relacionando ésta información con el medio y la teoría social existente sobre el problema estudiado. La construcción teórica es simultánea con la propia constitución del objeto de investigación.

A partir de lo anteriormente mencionado, surge entonces la siguiente pregunta: ¿Cómo son los estilos parentales de enseñanza utilizados en familias rurales con niños con necesidades educativas especiales del Colegio la Libertad del municipio de Samacá?; para dar respuesta a esta pregunta, se trazó el siguiente objetivo: indagar los estilos parentales de enseñanza utilizados en familias rurales con niños con necesidades educativas especiales del Colegio la Libertad del municipio de Samacá.

\section{METODOLOGÍA}

Esta investigación se abordó desde una visión cualitativa, desde el enfoque crítico-social y el diseño de la etnografía crítica. La investigación cualitativa es el tipo de investigación que intenta hacer una aproximación global de las situaciones de las ciencias sociales para describirlas y comprenderlas de manera inductiva, es decir, a partir de los conocimientos, experiencias, creencias y significados que tienen las diferentes personas involucradas en ellas (Bonilla \& Rodríguez, 1997).

El enfoque investigativo crítico-social, es definido como el enfoque que sirve al interés emancipatorio, hacia la libertad y la autonomía racional, procurando ofrecer a los individuos un medio para concienciarse de cómo sus objetivos y propósitos pueden haber resultado distorsionados o reprimidos y especificar como erradicarlos de manera que posibilite la búsqueda de las verdaderas metas. La misión de una ciencia social-crítica es disolver estas limitaciones haciendo que los mecanismos causales subyacentes sean visibles para aquellos a quienes afectan (Carr \& Kemmis, 1988).

La etnografía crítica, como el diseño asumido para esta investigación, pretende describir y analizar las ideas, creencias y prácticas de grupos, culturas o comunidades, (Atkinson \& Hammersley, 1994), además de describir e interpretar de manera profunda los cambios que se puedan dar en un grupo o sistema social (Creswell, 2009).

Para esta investigación se contó con 15 padres de familia de niños con necesidades educativas especiales del área rural, de la Institución Educativa Técnica "La libertad", del municipio de Samacá, Boyacá. Se contó con la participación de 12 madres en edades entre los 26 y 43 y 3 padres entre los 36 y 45 años de edad. Las necesidades educativas especiales identificadas en esta población son trastorno de aprendizaje y problemas de audición. El muestreo de esta investigación se hizo por medio del criterio lógico (Patton, 1998).

La recolección de la información se realizó mediante diferentes técnicas como la observación participante, el taller investigativo y la entrevista semiestructurada a grupo focal.

Sandoval (2002) define la técnica de la observación participante como aquella que surge como una alternativa distinta a las formas de observación convencional. Esta no es otra cosa que un registro continuo y acumulativo de todo lo acontecido durante la vida del proyecto de investigación. La observación participante emplea, para definir el problema de investigación con referencia a la vida cotidiana de las personas, una estrategia flexible de apertura y cierre. Esto quiere decir que puede comenzar con un problema general, para más tarde definir unos escenarios específicos de análisis.

El taller investigativo tiene como fortaleza principal brindar la posibilidad de abordar, desde una perspectiva integral y participativa, situaciones sociales que requieren algún cambio o desarrollo, siendo a su vez una estrategia de análisis y de 
planeación. Para este instrumento se debe tener en cuenta la operatividad y eficacia de la estrategia porque requiere un alto compromiso de los actores y una gran capacidad de convocatoria, animación y conducción de los investigadores (Sandoval, 1996).

La entrevista semiestructurada a grupo focal, se basa en una guía de asuntos o preguntas y el entrevistador tiene la libertad de introducir preguntas adicionales para precisar conceptos $\mathrm{u}$ obtener mayor información sobre los temas deseados (Hernández, Fernández \& Baptista, 2010). Esta técnica se centra en el abordaje a fondo de algún tipo de problema establecido, y se realiza a grupos de personas que poseen las mismas características, con el fin de obtener información ágil y pertinente que brinde la posibilidad de dar respuesta a corto plazo a las necesidades sociales que se investigan. La entrevista focal es semiestructurada, de aplicación colectiva y va enriqueciéndose y reorientándose conforme avanza el proceso investigativo (Bonilla \& Rodríguez, 1997).

Se utilizarán diferentes instrumentos para la recolección de información, tales como la bitácora o diario de campo, observación y relato de vida. Para el análisis de los datos cualitativos se utilizó el sistema categorial, que permitió la construcción y validación de categorías para la estructuración del estudio (Sandoval, 1996).

\section{Procedimiento}

Fase 1. Para el acercamiento con la población participante, se desarrolló un cuestionario que permitió identificar los datos sociodemográficos, el tipo de necesidad educativa especial de los niños y características propias del núcleo y el entorno familiar de los partícipantes. Posteriormente se determinaron y definieron los instrumentos de recolección de los datos a partir de las técnicas elegidas y la literatura revisada, estos instrumentos deben responder a las características de la investigación y lo que se pretende desarrollar en esta pesquisa.

Fase 2. Para esta fase de la investigación se implementaron 6 talleres investigativos. En primer lugar, se citaron a los padres de familia que han sido identificados con algún miembro en edad escolar con algún tipo de necesidad educativa especial. Antes de iniciar los talleres se les dio a conocer el consentimiento informado para la libre participación de estos padres en las actividades planteadas y los compromisos que ellos debieron asumir para el proceso investigativo. A nivel general, el diseño de los talleres tuvo la siguiente estructura, lo primero que se hizo en cada taller era formular la preocupación temática y la reflexión inicial sobre la situación, esto implica: reflexionar acerca de lo que se está haciendo en la actualidad sobre la temática a debatir, comprender los valores involucrados dentro del problema, el conocimiento de cómo encaja la problemática en términos de la experiencia que están viviendo los padres de familia y el conocimiento y comprensión de como se ha desarrollado el problema en la comunidad de padres de familia.

En esta fase también se desarrollaron los relatos de vida con los padres de familia con educativas especiales. Dentro de este relato de vida se formularon preguntas a los participantes con respecto a su experiencia personal frente a la los estilos parentales de enseñanza, pidiendo que relataran experiencias frente a los castigos y premios dados a sus hijos con de necesidades educativas especiales; en seguida, con el registro escrito elaborado por los participantes, se realizó la transcripción de los datos para su posterior análisis e interpretación.

Igualmente, en este proceso de la investigación se desarrolló una entrevista focal con los padres de familia que participaron del proceso, haciendo una convocatoria a una sesión única con los participantes que estaban interesados en participar y realizar la entrevista. Para ésta se realizó una guía de entrevista semiestructurada para preparar los temas generales sobre los estilos parentales de enseñanza que permitieron conducir la discusión, profundizar y ampliar los aspectos más relevantes sobre el tema. También se establecieron los roles de los investigadores (entrevistador y observador asistente) para una mejor recolección de la información. Para la toma de los datos de esta entrevista se grabaron 
en audio las diferentes respuestas de los padres de familia participantes.

Los datos, a medida que fueron emergiendo, fueron inmediatamente transcritos para su posterior sistematización y categorización.

Fase 4. Se realizó el análisis de los resultados obtenidos utilizando el sistema categorial o diseño sistemático propuesto por Sandoval (1996, p. 159). Por medio de este sistema de categorización, primero se realizó la codificación descriptiva y primer nivel de categorización; en esta fase se reunieron las unidades hermenéuticas correspondientes a cada una de las categorías de análisis o deductivas en la cual aparece un primer tipo de categorías descriptivas que emergen o surgen de un primer contacto con los datos recolectados. En segundo lugar se realizó la codificación axial o relacional: estas unidades hermenéuticas ya clasificadas, se subdividen internamente dentro de cada categoría deductiva, haciendo un proceso de conceptualización de los datos obtenidos, vinculando las categorías dos o más observaciones descriptivas entre sí, a partir de este nivel de categorización surgen las denominadas categorías inductivas que son de orden más teórico y vinculan entre sí dos o más categorías descriptivas o teóricas de orden inferior.

Finalmente se realizó la codificación selectiva y tercer nivel de categorización, en el cual se hace una depuración empírica y conceptual, que incluye la triangulación y la contrastación o feedback, que arrojará como resultado la identificación o el desarrollo de una o varias categorías núcleo, que articularán todo el sistema categorial construido a lo largo de la investigación.

Categorías de análisis o deductivas. Las categorías deductivas adoptadas para esta investigación surgieron a partir de la revisión teórica teniendo en cuenta los aportes de Baumrind (1966), y Maccoby y Martin (1983).

Estilo parental de enseñanza autoritario. Esta categoría define la forma en que los padres de familia ejercen el autoritarismo, el control y fijan reglas estrictas de comportamiento sobre los niños con necesidades educativas especiales los niños.

Estilo parental de enseñanza democrático. Esta categoría representa la forma en que los padres ayudan a sus hijos a valerse por sí mismos, tomando en cuenta sus apreciaciones. Este estilo se caracteriza por el desarrollo de la afectividad para la educación de los niños.

Estilo parental de enseñanza permisivo. Esta categoría describe la forma en que los padres ceden la mayoría del control a sus hijos. Este tipo de enseñanza se caracteriza por fijar muy pocas reglas, las cuales generalmente no se hacen cumplir de manera uniforme, además de que no se establecen límites ni tienen expectativas claras del comportamiento de sus hijos.

Estilo parental de enseñanza negligente. Esta categoría describe la forma en que los padres de familia ejercen poco control en sus hijos, establecen reglas básicas de comportamiento, evaden las responsabilidad de cuidado, son poco afectivos y consideran que ellos deben ser totalmente responsables de sus vidas, así si cometen algún error no es responsabilidad de ellos y no se generaran problemas, remplazan el afecto por cosas materiales y los hijos toman sus propias decisiones.

\section{RESULTADOS}

De los diferentes estilos parentales desarrollados por los padres de los niños con necesidades educativas especiales vinculados al colegio La Libertad del municipio de Samacá en Boyacá, a través de los resultados obtenidos, se puede afirmar que cuando los padres utilizan el estilo parental autoritario, éste se encuentra caracterizado por la intimidación de éstos hacia sus hijos, enfocándose en el uso del poder para imponer reglas y normas, poder que atribuyen desde su posición como padres, recurriendo de esta manera a castigos físicos y verbales para conseguir el control y corregir conductas; los castigos son ocasionados por la desobediencia, el incumplimiento en las exigencias de los padres, 
bajo rendimiento académico y la falta de apoyo en las actividades domésticas.

Así mismo, se evidenció que en las familias que desarrollan este estilo parental, la educación está establecida de manera monoparental autocrática, los cuidados del hogar y formación de los hijos son responsabilidad de las madres; en este tipo de familias el padre es visto como una figura de autoridad y de poder, siendo el encargado de impartir órdenes, estos padres imponen castigos a sus hijos por desacato a su autoridad, pero a la vez dan premios de acuerdo al cumplimiento de sus exigencias; dichos premios son mayoritariamente recompensas materiales y autorizaciones para realizar actividades lúdico-recreativas e interacción psicosocial.

En los resultados relacionados con el estilo parental autoritario se resalta la autoridad impuesta y arbitraria que los padres utilizan. Igualmente, se halló que las familias perciben que la crianza de los hijos debe ser dirigida única y exclusivamente por los padres, puesto que ellos son los responsables de formar personas capaces y con un alto sentido del liderazgo, obedientes, respetuosos e inteligentes; se deja de lado las muestras de cariño y expresiones de afecto, considerando que esto puede generar malos comportamientos en los hijos. La mayoría de los padres de familia recurren a una forma de educación fundamentada en premios y castigos, alta exigencia y poca expresión afectiva.

En cuanto al estilo parental permisivo de los padres de niños con necesidades educativas especiales, se pudo establecer que cuando los padres implementan este tipo de educación con sus hijos, se caracterizan por la alta afectividad, pero así mismo se presentan como padres indulgentes, debido a que no establecen normas de conducta en el hogar y son flexibles ante las exigencias de los hijos, por lo que generalmente estos niños tienen la libertad para expresarse y para realizar lo que deseen. De este modo los padres permisivos comúnmente evaden al máximo imponer castigos por miedo que sus hijos tengan una reacción negativa ante ellos, son padres que presentan altos niveles de condescendencia y permisividad ante los comportamientos de los menores, limitándose a dar refuerzos por conductas apropiadas, y evadiendo la responsabilidad de guiar a los niños cuando tienen actitudes negativas justificándose en el pleno desarrollo de la autonomía de sus hijos. Un aspecto positivo encontrado en este estudio con los padres que manejan este estilo de enseñanza, sumado al alto grado de afectividad, es que entre estos se da una comunicación adecuada.

Así mismo, dentro del desarrollo de este estilo parental de enseñanza, los padres optan por no proporcionar castigos a sus hijos aun cuando los niños presentan comportamientos inadecuados, esto con el fin de que los hijos no tengan una percepción errada acerca del amor que tienen los padres hacia ellos, debido a que se manejan creencias erradas respecto al castigo, viéndolo como una acción negativa en la formación de los hijos.

En el estilo parental negligente, dentro de la población estudiada, se describe inicialmente por la irresponsabilidad de la figura masculina en el cuidado y el apoyo de los hijos en el hogar; los padres que acogen este estilo parental son poco afectivos, no tienen una comunicación directa con sus hijos, además no establecen normas ni reglas de convivencia que regule la conducta de los hijos. Este tipo de padres demuestran poco interés en las demandas de los niños, por lo que estos tienen absoluta libertad de hacer lo que ellos deseen, así mismo dentro de este tipo de familias se evidencia que las madres son las únicas encargadas de las labores domésticas y el cuidado y formación escolar de los hijos, los padres desempeñan exclusivamente una función de autoridad, debido a que estos propician a sus hijos castigos físicos con el agravante de hacerlo bajo estado de embriaguez y sin ninguna justificación. El tiempo que este tipo de padres dedica a sus hijos es casi nulo, dado que dan prioridad a otras actividades como las laborales y actividades sociales con personas externas de la familia. Es necesario resaltar que se evidenció que estos padres muestran ignorancia ante el apoyo en el desarrollo de actividades escolares de sus hijos por diversos motivos como la baja escolaridad y en algunos casos, el analfabetismo. 
Los padres que adoptan el estilo parental democrático, construyen una crianza basada en valores (amor, respeto, tolerancia y comprensión), con control y exigencias, estableciendo normas y reglas de convivencias, teniendo en cuenta que estos padres son más flexibles ante las necesidades y opiniones de los hijos; además brindan apoyo, comprensión y afectividad a los niños, con el fin de que estos sean emocionalmente estables, seguros, independientes, obedientes. Los padres democráticos también imponen a sus hijos castigos y brindan recompensas, pero estos son razonados, además que cuando se deben aplicar se explica a los niños por qué se va a dar una sanción o un refuerzo.

Los castigos de estos padres se basan en prohibiciones de actividades placenteras e inhibición de autorizaciones para interactuar con la familia extensa y contacto social más prolongado, además de utilizar el dialogo como medio de resolución de conflictos; los niños son castigados con el deseo de eliminar o disminuir conductas inapropiadas establecidas en familia; las recompensas que se les otorgan a los hijos se hacen a través de expresiones y palabras afectivas, alimentos apetitivos, actividades lúdico-recreativas en familia o en algunas ocasiones son materiales (dinero, detalles); dichas recompensas se conceden ante el buen comportamiento de los hijos en el hogar, buenos resultados académicos, atención ante las exigencias de éstos. Los representantes de este estilo parental utilizan un estilo comunicativo bidireccional que les permite fomentar la confianza en el hogar, además les permite apoyar a los hijos en las actividades académicas para que sean realizadas con éxito, dando instrucciones verbales adecuadas.

Teniendo en cuenta lo encontrado en la población de estudio, se evidencia que los padres democráticos mantienen niveles de exigencia y control sobre sus hijos, pero estos dan la oportunidad a los niños de vincularse en la toma de decisiones al momento de establecer normas de conducta, además estos padres a pesar de ser exigentes basan su crianza en valores como el amor y el respeto.

\section{DISCUSIÓN}

Frente al estilo parental autoritario se puede analizar en relación a los resultados, que éste estilo de enseñanza basado en la imposición de normas y reglas para mantener el control absoluto de los comportamientos de los infantes, deja de lado las necesidades principales de los hijos. Este estilo educativo de los padres de familia, no permite establecer estrategias de comunicación que permitan la resolución de conflictos de forma adecuada. Por estos motivos, la autoridad e imposición de normas inquebrantables de los padres hace que los hijos de familias autoritarias sean niños inseguros, con poca auto confianza, miedo a expresarse y que carezcan de habilidades sociales, básicamente los convierten en niños dependientes y con una autoestima baja. Como refieren Olson, Sprenkle y Russell (1979), los padres que adoptan este estilo parental, ejercen el control unidireccionalmente sin contar con la opinión de los hijos, así mismo se muestran muy exigentes y tienen poca sensibilidad ante las demandas de éstos a quiénes no les dejan participar ni mostrar su opinión utilizando la imposición para establecer su criterio y castigando con dureza las faltas.

Se considera entonces que estas pautas represivas y centradas solamente en las figuras paternas, perjudican el comportamiento y desarrollo íntegro del niño como una persona capaz de desenvolverse correctamente en su contexto, además de afectar la forma en que el menor con dificultades del aprendizaje pueda llegar a relacionarse de forma eficaz en todas sus esferas de funcionamiento, puesto que se observa que estos niños presentan conductas mecánicas, es decir, no tienen autonomía ni criterio para actuar de cierto modo, esperan la orden y el consentimiento de la autoridad antes de cualquier acción.

Cómo lo mencionan Esteves, Jiménez y Musito (2007), los niños que son educados bajo el estilo parental autoritario presentan más problemas para tener relaciones adecuadas con los demás, utilizan maneras poco adecuadas para enfrentar los conflictos, no tienen buenos resultados 
académicos, presentan más problemas de integración escolar y baja autoestima.

Por último, se puede interpretar que estas pautas de crianza poco asertivas se centran en proyectar lo sufrido por los padres de familia en la niñez y que ahora es considerado como lo "correcto" para educar a los niños. Sin duda esta situación deja ver la falta de actualización y educación en las zonas rurales en aspectos determinantes de la crianza de los niños. Tal como lo plantean Palacios y Rodrigo (1998), las prácticas educativas de los padres pueden estar determinadas por una serie de factores como lo son el sexo, las experiencias previas vividas como hijos y como padres, características de personalidad desarrollada por la crianza recibida, nivel educativo, ideas acerca del proceso evolutivo y la educación y expectativas de logros que tienen puestas en sus hijos.

Frente al estilo parental permisivo se puede analizar desde los resultados obtenidos, que éste estilo de crianza evidencia la falta de autoridad, como refieren Del Pozo, Cabanyes y Polaino (2003), los padres permisivos no ejercen ningún control sobre sus hijos, y su nivel de exigencia es muy bajo, estos padres suelen ser muy afectuosos, permiten casi todo a sus hijos, lo que puede condicionar que estos se manifiesten como niños inseguros y afectivamente dependientes.

De esto se puede interpretar que la evitación del castigo en el proceso de educación, genera en los niños conductas inapropiadas donde prevalece la falta de respeto ante figuras de autoridad y hacia sus pares, debido a que estos niños no conocen las consecuencias que tienen los malos comportamientos, puesto que sus padres no utilizan correctivos frente a sus malas acciones. De acuerdo con Baumrind, (1966) la dificultad del estilo parental permisivo, es principalmente por el afecto de los padres hacia los hijos y de igual forma por la ausencia de control o restricciones, lo que se traducen en una actitud general positiva de los padres hacia el comportamiento de sus hijos, estos aceptan las conductas de los niños y utilizan muy poco el castigo, evitan recurrir a la autoridad y dejando de lado las exigencias, proporcionan toda la autonomía posible y dejan que sus hijos que decidan por sí solos.

De este modo se considera que la inhibición del castigo y la poca autoridad de los padres generan que estos niños sean mal criados, desobedientes, imprudentes y manipuladores. Reynaud y Sanchez (1996), refieren que la ausencia de limites por parte de estos padres, impide que los niños adquieran habilidades de autocontrol, los hijos de padres de este estilo parental tienden a ser inmaduros, no controlan sus impulsos, ni son socialmente responsables, además muestran una menor capacidad intelectual; por lo tanto estos padres permiten que sus hijos actúen impulsivamente y no demandan una conducta madura por parte de ellos.

Frente al estilo parental negligente, se puede interpretar que en el desarrollo de esta forma de enseñanza de los padres de familia, disminuye la probabilidad de generar relaciones de apego con sus hijos, puesto que estos establecen como prioridad las obligaciones laborales, la interacción social y el consumo de bebidas alcohólicas, por lo tanto no tiene un espacio para compartir con su familia, trasmitiendo a sus hijos sentimientos de rechazo, abandono y poca afectividad. Como lo afirman Maccoby y Martin (1983), el estilo de crianza negligente es el utilizado por padres que rechazan, que no son receptivos ni exigentes y a quienes sus hijos parecen serles indiferentes. A estos niños se les da tan poco como se les exige, pues los padres presentan unos niveles muy bajos en las cuatro dimensiones: afecto, comunicación, control y exigencia, en este estilo no hay normas que cumplir ni afecto para compartir, siendo lo más destacable la escasa intensidad de los apegos y la relativa indiferencia respecto a las conductas del niño.

Así mismo, se puede analizar que el escaso apoyo e interés de los padres hacia sus hijos propicia que estos sean inseguros, agresivos, con pocas habilidades para relacionarse con otros, baja autoestima, poco autocontrol y un errado concepto acerca de la familia, además de tener poco 
éxito en las actividades escolares (rendimiento académico bajo). Esteves, et al. (2007), refieren que los hijos de padres negligentes suelen tener problemas psicológicos como ansiedad, depresión, baja autoestima y falta de empatía, así mismo tienen problemas de comportamientos (conductas agresivas) y son menos competentes para establecer relaciones con los demás.

Frente al estilo parental democrático se puede considerar que el desarrollo de este estilo de enseñanza en la familia, permite que los padres sean más conscientes sobre lo que deben corregir a sus hijos pero al mismo tiempo necesitan desarrollar estrategias que les permita castigar sin necesidad de recurrir agresiones físicas o verbales. Los padres de familia que implementan estos estilos parentales construyen relaciones afectivas flexibles, democráticas y tolerantes, pero al mismo tiempo estableciendo límites claros. Estos padres permiten que sus hijos opinen y les colaboren con cariño en las actividades familiares, además logran mezclar adecuadamente la disciplina con el amor. Los resultados evolutivos en competencias cognitivas y sociales de este estilo ubican a los niños en un rendimiento académico, habilidades sociales, autoestima y preocupaciones morales bastante altas (Maccoby \& Martin, 1983).

La comunicación bidireccional dentro de este estilo parental de enseñanza, brinda la oportunidad a los padres de dar explicaciones a los hijos cuando es necesario respecto a sus comportamientos, facilitando las expresiones afectivas en la familia, además de realimentar las acciones de los niños respecto a un castigo o una recompensa, lo que permite que los hijos de este estilo de padres conozca cada una de sus faltas y sus logros, lo que beneficia la autonomía de estos. Tal Como lo indica Baumrind (1966), los padres con estilo democrático tienen confianza en ellos mismos como padres y como personas, por lo que con seguridad establecen y comunican pautas de conductas para que sus hijos sigan tomando en cuenta sus necesidades, tanto de los iguales como de la sociedad; de igual forma en la toma de decisiones los hijos tienen una parte importante, lo cual proporciona una buena actitud de dialogo en la familia, en este sentido la última decisión corresponde a los padres pero no sin antes haber analizado la postura de los demás miembros de la familia.

\section{CONCLUSIÓN}

El estilo parental autoritario se describe por un excesivo control y exigencia de los padres, además de la implementación de castigos físicos y verbales y el uso de recompensas materiales con el fin de mantener o disminuir conductas en los hijos, según las normas establecidas en el hogar.

El uso del estilo autoritario es un problema cultural, debido a que la mujer es vista como la única responsable del cuidado del hogar y de la educación de los hijos y el padre tiene como única función desempeñar actividades laborales fuera del hogar, además de reforzar los castigos impuestos por las madres; de igual forma también se evidencia la falta de comunicación que existe entre los padres, debido al poco tiempo que utilizan para comunicarse entre ellos y con sus hijos. Los padres autoritarios ven este estilo parental como la forma ideal para educar a los niños con necesidades educativas especiales.

El estilo parental permisivo se caracteriza por el alto grado de afectividad y condescendencia de los padres hacia los hijos. Los padres permisivos son altamente flexibles ante las exigencias y acciones de los hijos, generando en ellos autonomía para realizar cualquier tipo de actividad, de igual forma estos niños son incapaces de seguir órdenes y respetar a las figuras de autoridad.

El estilo parental negligente está representado por el rechazo y abandono de los padres frente al cuidado y formación de los hijos, los padres negligentes no establecen como prioridad a los hijos sino a las actividades laborales y de interacción social, dejando el cuidado de los hijos a terceras personas. En la población estudiada, el estilo parental es característico de la figura masculina en el hogar, por lo que el cuidado y educación de los hijos es responsabilidad únicamente de las madres, debido a esto los 
padres son vistos como una figura de autoridad que solo propicia castigos físicos y brinda apoyo económico para la familia.

El estilo parental democrático se caracteriza por las exigencias y control que ejercen los padres, siendo estos al mismo tiempo afectivos y comprensivos. El estilo parental democrático es el más asertivo para la crianza de los hijos, puesto que en estos hogares maneja un alto niveles de confianza y comprensión, lo que genere que los hijos de este tipo de padres sean niños autónomos, seguros de sí mismos, con alta autoestima, independientes y capaces de desenvolverse en diferentes entornos.

En las familias participantes no prevalece un mismo estilo parental entre los padres, debido a que se evidencia que las madres son las encargadas del cuidado de los hijos y del hogar, mientras que en gran parte la figura masculina es representada como negligente, teniendo en cuenta lo referido por las madres participantes donde se devela la ausencia de la figura paterna en el hogar.

En general, se puede analizar que el estilo parental más utilizado por los padres dentro de la población de estudio es el autoritario, debido a que se da por modelamiento de los padres respecto a las experiencias vividas en su infancia.

\section{REFERENCIAS BIBLIOGRÁFICAS}

Aguirre, E. (2000). Socialización y prácticas de crianza. En E. Aguirre y E. Durán. Socialización: Prácticas de Crianza y cuidado de la salud. Bogotá, D. C.: CES- Universidad Nacional de Colombia.

Aranda, I. (2009). Las necesidades educativas especiales. La relación escuela- familia- comunidad. Cuadernos de Educación y Desarrollo. Vol 1, Nº 7 Malaga, España.

Atkinson, P., \& Hammersley, M. (1994). Etnografía: métodos de investigación. Barcelona, España.

Avila, L., Malagon, A., \& Triana, A. (2010). Prácticas de crianza y cuidados en niños y niñas de en Boyacá. Revista Latinoamérica de ciencias sociales y niñez, 8 (2): 933 - 945.

Baumrind, D. (1966). Effects of authoritative control on child behaviour. Child Development, 37, 887-907.
Bonilla, E., \& Rodríguez, P. (1997). Más allá del dilema de los métodos: la investigación en ciencias sociales. Editorial norma. Bogotá, Colombia.

Borke, J., Lamm, B., Eickhorst, A., \& Keller, H. (2007). Father-infant inte-raction, paternal ideas about early child care, and their consequences for the development of children's self-recognition. The Journal of Genetic Psychology. 168; 365-379.

Carr, W., \& Kemmis, S. (1988). Teoría crítica de la enseñanza. La investigación-acción en la formación del profesorado. Barcelona, España.

Ceballos, E \& Rodrigo, M. (1998). Las metas y estrategias de socialización entre padres e hijos. En Rodrigo, M \& Palacios, J (Ed). Familia y desarrollo humano, Madrid: Alianza.

Creswell, J. W. (2009). Research Design: qualitative, quantitative, and mixed methods approaches $\left(3 .^{\mathrm{a}}\right.$ ed.). Londres: Sage.

Del Pozo, A., Cabanyes, J., \& Polaino, A. (2003). Fundamentos de psicología de la personalidad. Navarra, España.

Esteves, E., Jiménez, T., \& Musito, G. (2007). Relación entre padres e hijos adolescentes. Editorial Nau Llibres. Valencia.

Hernandez, Fernandez \& Baptista. (2010). Metodología de la investigación. McGRAW-HILL. Ed. S.A de C.V.

Maccoby, E. \& Martin, A. (1983). Socialization in the context of the family: parent-child interactions. E.M. Hetherington \& P.H. Mussen (eds.). Socialization, personality and social development. Handbook of child psychology, Vol. IV. Cap. 9: 1-102. New York: Wiley.

Myers, R. (1994). Prácticas de crianza. Bogotá: CELAM-UNICEF.

Myers, R. (1994). Prácticas de crianza. Bogotá: CELAM-UNICEF.

Olson, D. H., Sprenkle, D. H., \& Russell, C. S. (1979). Circumplex model of marital and family systems: I. Cohesion and adaptability dimensions, family types and clinical applications. Family Process, 18, 3-28.

Palacios, J., \& Rodrigo, M. J. (1998). La familia como contexto de desarrollo humano. En M. J. Rodrigo \& J. Palacios (coords.), Familia y desarrollo humano, 25-38. Madrid: Alianza Editorial.

Patton, M. Q. (1988). How to Use Qualitative Methods in Evaluation. Newbury Park-California: Sage.

Reynaud, B., \& Sanchez, J. (1996). Psicología y problemática social. México.

Sandoval, C. (1996). Programa de especialización en teoría, métodos y técnicas de investigación social, investigación cualitativa. Bogotá. Arfo Editores e Impresores Ltda.

Sandoval, C. (2002). Investigación Cualitativa. Editores e Impresores. Bogotá, Colombia. 